\title{
Solvent effect in the theoretical absorption and emission spectra of fluorescein dyes
}

\author{
F. N. Tomilin*a,b, A. V. Rogova ${ }^{a}$, E. V. Kaufman ${ }^{a}$, \\ A. S. Drevolsky, M. A. Gerasimova ${ }^{a}$, and E. A. Slyusareva ${ }^{a}$ \\ ${ }^{a}$ Siberian Federal University, Svobodny Prospect 79, Krasnoyarsk, 660041 Russia \\ ${ }^{b}$ Kirensky Institute of Physics, Krasnoyarsk Scientific Center, Siberian Branch, Russian Academy of \\ Sciences, Akademgorodok 50, bld. 38, Krasnoyarsk, 660036 Russia
}

\begin{abstract}
Fluorescein and its halogenated derivatives representing a family of homologous dyes with the gradual substitution of halogen atoms for hydrogen ones are widely used in biomedicine as fluorescent probes. This stimulates the intense experimental and theoretical studies of their fluorescent properties in aqueous solutions. However, the theoretical calculations are complicated by the necessity of taking into account the effect of a solvent (water) in the explicit form and the need for effective basic sets. This is especially important for the dyes that contain heavy atoms. In this study, the quantum-chemical investigations of the dianionic form of fluorescein and its Br-and I-substituted derivatives (eosin Y and erythrosin B) have been carried out using the time-dependent density functional theory (B3LYP functional) implemented in the GAMESS software suite. The effect of a solvent has been considered in the framework of the modified Thomas polarizable continuum model. The calculations have been made for vertical (absorption and emission) excitations in the adiabatic approximation and at the nonequilibrium solvation. The results obtained for the nonequilibrium solvation are in excellent agreement with the experimental data for fluorescein and its halogenated derivatives.
\end{abstract}

Keywords: fluorescein, eosin Y, erythrosin B, dianion, PCM, time-dependent density functional theory

\section{INTRODUCTION}

Fluorescein and its halogenated derivatives (eosin Y, phloxine B, erythrosin B, rose Bengal, and others) are well-known fluorescent probes [1-3]. The high absorbance and fluorescent properties of the fluorescein-based dyes make them good candidates for use in marking and visualizing colorless systems, including living cells, proteins, and carbon nanotubes. The possible direction in the development of biosensor applications of the dyes is more thorough investigations of their fluorescent properties, since the latter bear imprints of high-speed processes due to the short lifetime of the excited state.

Such high-speed processes include the processes with a solvent involved. It is well-known that the medium relaxation mechanisms (molecular reorientation, atomic vibrations, and electronic polarization of relaxation of a medium) are fairly complex and their characteristic times lie in different ranges. The correct account for a polar solvent in building quantummechanical models is a challenging problem. To solve it, the solvent molecules are taken into account in the explicit or implicit form. In the former case, a finite number of solvent molecules are taken into account along with the investigated molecule and, in the latter case [4], a solvent is considered to be an infinite continuum with certain dielectric properties [5]. The implicit account for a solvent allows one to take into account different mechanisms of relaxation of a medium [6].

In this study, we investigate dianions of fluorescein and its Br- and I-substituted derivatives (eosin $\mathrm{Y}$ and erythrosin $\mathrm{B}$ ) dissolved in water using the quantum-mechanical calculations (B3LYP) by different techniques for taking a solvent into account and discuss the geometry and distribution of molecular charges. The efficiency of different solvent accounting techniques is analyzed by comparing the results of the calculation with the experimental absorption and emission data.

\section{METHODS}

\subsection{Experiment details}

Sodium salts of fluorescein (Fluka), eosin Y (Sigma-Aldrich) and erythrosin B (Aldrich) were used without additional purification. The dianionic form of the dyes in a concentration of $5 \mu \mathrm{M}$ in aqueous solution with $\mathrm{pH} 11$ was prepared. The 
absorption spectra were recorded on a PerkinElmer Lambda 35 spectrophotometer. The fluorescence spectra were recorded on a Horiba Jobin Yvon Fluorolog 3-22 spectrofluorometer and corrected for the reabsorption and sensitivity of the recording systems. Standard quartz cells $10 \times 10 \mathrm{~mm}$ in size were used. All the measurements were performed at room temperature.

\subsection{Computation details}

In all the computations, the B3LYP theory level was used [7, 8]. Two types of the calculations by the time-dependent density functional theory $[9,10]$ with a polarizable continuum model (TD-DFT/PCM) were made using the GAMESS program suite [11]. In the type-one (PCM) calculations [4], the electron energies and oscillator strengths were computed for a permittivity of $\varepsilon=78.39$, assuming the solvent relaxation in the ground and excited states by setting $N O N E Q=. F$. in the GAMESS software. In the type-one ( $\left.\mathrm{PCM}^{*}\right)$ calculations [6], the nonequilibrium vertical energies and oscillator strengths were computed for a permittivity of $\varepsilon_{\infty}=1.776$ by setting NONEQ=.T. in the GAMESS software. The fluorescein dianion geometry was fully optimized for the ground state with no symmetry constraints, separately in the vapor phase and in the solution. Then, the vertical excitation energies for the absorption spectra were calculated as differences between the energies of the excited and ground states, both computed in the ground state geometries. The emission energies were determined as the vertical de-excitation energies corresponding to the transitions between the excited and ground states in the excited state geometries.

\section{RESULTS AND DISCUSSION}

\subsection{Calculation models}

In this study, the solvent was taken into account using the polarizable continuum model (PCM). This model is grounded on separating a solute (the molecule can be described at the desired theory level) and a solvent in the form of a structureless infinite continuum characterized by its own macroscopic permittivity and density. The interface between a solute and a solvent is presented in the form of a closed surface built around the solute molecule. The permittivity $\varepsilon$ inside the cavity formed by the surface is unity, i. e., is equal to the permittivity of vacuum, while the permittivity outside the cavity is equal to the permittivity of the investigated solvent (e. g., 78.4 for water at $25^{\circ} \mathrm{C}$ ). In describing the dynamic processes, the molecule is specified using another approach. The shape of a molecule of a real solute is imitated by building a cavity in the form of a spherical envelope located on dissolved atoms or atomic groups. To level the surface, other spheres can be added in the so-called solvent-excluded surface approximation. The procedure of forming a cavity can be continuously refined. The important parameters are radii of atomic spheres, which are optimized to ensure the solvation free energies similar to the experimental values for a great set of inorganic and organic molecules. In this study, the dyes were calculated using both the conventional PCM and nonequilibrium PCM* solvation methods [6] and the effect of the solvent relaxation time on the absorption and emission spectra of the fluorescein dyes was examined. The solvent relaxation time is taken into account in the case of absorption or emission of photons with the subsequent electronic transition, when the electron density of solute charges rapidly changes. It means that only solvent electrons can move to stay in equilibrium with the solute, while the molecular motion of a solvent during the process is frozen.

As a rule, with an increase in the basis set, the calculation characteristics (atomic structure, absorption and emission wavelengths, etc.) are improved and approach their experimental values [12]. The similar trend is observed at the transition from the vapor phase to the models that take the solvent into account (PCM, $\mathrm{PCM}^{*}$ ). In this case, the sharp (by up to 42 $\mathrm{nm}$ ) growth of the calculated values and their similarity to the experimental data are obtained when the solvent relaxation is taken into account $\left(\mathrm{PCM}^{*}\right)$; therefore, the calculations show the importance of the «diffuse» functions used in simulating ions, excited states, and weak couplings. In a fluorescein dianion, this effect is especially pronounced at the transition from the $6-31^{*}$ to $6-311^{* *++}$ basis (the improvement by 6, 7, and $10 \mathrm{~nm}$ for vacuum, PCM, and PCM*, respectively) and at the transition from cc-pVDZ to aug-cc-pVDZ (the improvement by 8, 9, and $13 \mathrm{~nm}$ for vacuum, PCM, and PCM*, respectively). In this case, a simple increase in the basis functions from 6-31* to 6-311** and from aug-cc-pVDZ to augcc-pVTZ almost does not improve the result of the calculation. In other words, at the transition from 6-31** to 6-311**, the improvement for vacuum, PCM, and PCM* is merely by 1,2, and $3 \mathrm{~nm}$. The aforesaid is indicative of the fact that the combination for the quantum-mechanical calculation is the choice of an appropriate basis and a relevant solvent model. Basing on the preliminary calculations, the aug-cc-pVDZ basis was chosen for fluorescein dyes (with the exception of erythrosin B, for which the ADZP basis was chosen), which yields good results for reasonable time.

In addition, in the analysis of the calculated data, the parameter $\Lambda$ [13] was considered, which serves as a criterion for separating the local transitions from the transitions with charge transfer and from the Rydberg transitions. The small $\Lambda$ 
value is indicative of the long-distance excitation and the large $\Lambda$ value, of the short-distance one. Local excitations have a relatively large overlap $(0.45<\Lambda<0.89)$, which points out that both the occupied and virtual orbitals involved in the excitation hold analogous spatial regions. The same can be expected for the local excitations with the small degree of redistribution. At all the transitions, the parameter $\Lambda$ lied between $0.64<\Lambda<0.67$ for all structures. This can be indicative of the fact that the charge transfer play no meaningful role in dianions of the fluorescein dyes upon absorption and emission and the DFT functional like B3LYP can therefore be used.

\subsection{Analysis of the simulated absorption and emission spectra}

To analyze the electronic properties, partial charges on different parts of the fluorescein dyes were counted by summing hydrogen, oxygen, halogen, and carbon atoms contained in the corresponding parts of molecules. The dye dianion can be schematically divided into four rings (see the figure and Table 1). The monocycle is ring 1 and the tricycle consists of rings 2,3 , and 4 . According to the results of the calculation, dianions of the fluorescein dyes in the ground state can be presented in the form of a single spatial conformer, when the carboxyl group $\mathrm{COO}^{-}$lies in the monocycle plane. In this case, the monocycle plane remains almost perpendicular to the tricycle plane. The substitution of $\mathrm{Br}$ and I ions for hydrogen ones leads to a slight redistribution of partial charges. At the transition from fluorescein $\left(-0.12 e^{-}\right)$to erythrosin $\mathrm{B}\left(-0.11 e^{-}\right)$, the charge on oxygen in ring 3 weakens. The oxygen charges on rings 2 and 4 are similar for all the dyes; they are $-0.20 e^{-}$for fluorescein, $-0.18 e^{-}$for eosin $\mathrm{Y}$, and $-0.18 e^{-}$for erythrosin $\mathrm{B}$. The effect of the solvent on the charges of oxygen atoms consists in a slight increase in the negative partial charge in the range of $0.04-0.09 e^{-}$on oxygen atoms in rings 2,3 , and 4 , while the charge on oxygen in ring 1 remains almost unchanged. The substitution of $\mathrm{Br}$ and I atoms for hydrogen causes a slight redistribution of partial charges.

Table 1. Löwdin charges ( $e^{-}$) on the rings 1, 2, 3 and 4 (denoted in the structure) in the ground state of fluorescein dyes obtained at the B3LYP/aug-cc-pVDZ/PCM level of theory.

\begin{tabular}{|c|c|c|c|c|c|}
\hline & & & & & \\
\hline & Triple ring & & Indir & & \\
\hline & $2+3+4$ & 1 & 2 & 3 & 4 \\
\hline Fluorescein & -1.16 & -0.85 & -0.38 & -0.40 & -0.38 \\
\hline Eosin $Y$ & -1.21 & -0.79 & -0.36 & -0.49 & -0.36 \\
\hline Erythrosin $\mathrm{B}^{a}$ & -1.21 & -0.80 & -0.34 & -0.53 & -0.34 \\
\hline
\end{tabular}

${ }^{a}$ For erythrosin B the B3LYP/ADZP/PCM level of theory was taken.

If the charge increases on the chromophore group of atoms, then the change in the charge properties should affect the absorption and fluorescence spectra. It can be seen from Table 1 that fluorescein sharply differs from eosin Y and erythrosin $\mathrm{B}$, while the latter are very similar in the electronic properties. Upon the halogen substitution of bromine and iodine, the partial charge on ring 3 changes from $-0.40 e^{-}$to $-0.49-0.53 e^{-}$. In this case, the charge on the tricycle grows from $-1.16 e^{-}$ to $-1.21 e^{-}$, while the charge on monocycle 1 , vice versa, decreases from $-0.85 e^{-}$to $-0.80 e^{-}$. Such an increase in the charge on the tricycle should lead to a noticeable red shift in the halogenated fluorescein derivatives. Eosin Y and erythrosin B should have similar spectral properties.

Table 2 gives the calculated excitation (emission) wavelengths for all calculated dianions under the equilibrium (PCM) and nonequilibrium $\left(\mathrm{PCM}^{*}\right)$ solvation together with the experimental data. Generally, the Stokes shift calculated with regard to the equilibrium/nonequilibrium solvation agrees well with the experiment.

The shift of the absorption spectrum to the red region relative to the molecule calculated under the vapor phase conditions (e. g., for the fluorescein dianion, $\lambda_{\mathrm{abs}}=421 \mathrm{~nm} \mathrm{TD} / \mathrm{B} 3 \mathrm{LYP} / 6-31^{*}$ ) is affected by the calculation basis set and the account for a medium and solvent relaxation (Table 2). Adding the account for the nonequilibrium solvation to the «good» basis yields the best result. The absorption wavelength $\lambda_{\text {abs }}$ of the fluorescein dianion improved by 62 nm relative to the vapor 
phase and by $40 \mathrm{~nm}$ relative to the PCM calculation. This trend is observed for all calculated dianions. The difference was $36 \mathrm{~nm}$ for eosin $\mathrm{Y}$ and $34 \mathrm{~nm}$ for erythrosin B. Thus, for these dyes, the account for the nonequilibrium solvation is of great importance.

Table 2. TD-DFT properties of fluorescein dyes obtained at the B3LYP/aug-cc-pVDZ level of theory.

\begin{tabular}{|c|c|c|c|c|c|c|c|c|c|}
\hline & \multicolumn{3}{|c|}{ Fluorescein } & \multicolumn{3}{|c|}{ Eosin $Y$} & \multicolumn{3}{|c|}{ Erythrosin $\mathrm{B}^{a}$} \\
\hline \multicolumn{10}{|c|}{ Absorption } \\
\hline & $\lambda_{\mathrm{abs}}{ }^{b}, \mathrm{~nm}$ & $f_{\text {abs }}{ }^{c}$ & $\Lambda^{d}$ & $\lambda_{\mathrm{abs}}, \mathrm{nm}$ & $f_{\text {abs }}$ & $\Lambda$ & $\lambda_{\mathrm{abs}}, \mathrm{nm}$ & $f_{\text {abs }}$ & $\Lambda$ \\
\hline $\mathrm{PCM}$ & 443 & 0.80 & 0.67 & 458 & 0.88 & 0.65 & 464 & 0.83 & 0.64 \\
\hline PCM* & 483 & 1.16 & 0.67 & 493 & 1.20 & 0.65 & 498 & 1.21 & 0.65 \\
\hline $\mathrm{PCM}+4 \mathrm{H}_{2} \mathrm{O}$ & 441 & 0.83 & 0.67 & 460 & 0.83 & 0.65 & 464 & 0.82 & 0.64 \\
\hline $\mathrm{PCM}^{*}+4 \mathrm{H}_{2} \mathrm{O}$ & 479 & 1.15 & 0.67 & 493 & 1.18 & 0.66 & 498 & 1.15 & 0.65 \\
\hline Experiment & 490 & & & 516 & & & 526 & & \\
\hline \multicolumn{10}{|c|}{ Fluorescence } \\
\hline & $\lambda_{\mathrm{fl}}{ }^{e}, \mathrm{~nm}$ & $f_{\mathrm{fl}}^{f}$ & $\Lambda$ & $\lambda_{\mathrm{fl}}, \mathrm{nm}$ & $f_{\mathrm{fl}}$ & $\Lambda$ & $\lambda_{\mathrm{fl}}, \mathrm{nm}$ & $f_{\mathrm{fl}}$ & $\Lambda$ \\
\hline PCM & 466 & 0.71 & 0.66 & 473 & 0.80 & 0.65 & 486 & 0.75 & 0.65 \\
\hline PCM* & 509 & 1.05 & 0.67 & 511 & 1.17 & 0.66 & 522 & 1.13 & 0.65 \\
\hline Experiment & 513 & & & 536 & & & 547 & & \\
\hline \multicolumn{10}{|c|}{ Stokes shift, $\mathrm{nm}$} \\
\hline Theory/PCM & \multicolumn{3}{|c|}{23} & \multicolumn{3}{|c|}{15} & \multicolumn{3}{|c|}{21} \\
\hline Theory/PCM* & \multicolumn{3}{|c|}{26} & \multicolumn{3}{|c|}{18} & \multicolumn{3}{|c|}{24} \\
\hline Experiment & \multicolumn{3}{|c|}{23} & \multicolumn{3}{|c|}{20} & \multicolumn{3}{|c|}{21} \\
\hline
\end{tabular}

${ }^{a}$ For erythrosin B the B3LYP/ADZP level of theory was taken; ${ }^{b} \lambda_{\text {abs }}$ and ${ }^{c} f_{\text {abs }}$ are the absorption wavelength and oscillator strength of $\mathrm{S}_{0} \rightarrow \mathrm{S}_{1}$ transition, respectively; ${ }^{d} \Lambda$ is lambda parameter; ${ }^{e} \lambda_{\mathrm{fl}}$ and ${ }^{f} f_{\mathrm{fl}}$ are the fluorescence wavelength and oscillator strength of $\mathrm{S}_{1} \rightarrow \mathrm{S}_{0}$ transition, respectively.

As a rule, under the stationary conditions, the $\varepsilon$ values that only depend on temperature and pressure are used. However, in studying the dynamic properties, it is necessary to take into account the time of relaxation of the solvent polarization. If the characteristic time of the investigated process is shorter than the solvent relaxation time, then the response of the solvent is delayed. This can be described by the term of «fast» permittivity $\varepsilon_{\mathrm{f}}$, which is in equilibrium with a solute, whereas the remainder is delayed or even frozen if the electron density of a solute changes very fast. The «fast» permittivity depends on the mechanism of relaxation of a solvent (rearrangement of molecules, atomic vibrations, electron polarization, etc.). It is determined as

$$
\varepsilon_{\mathrm{f}}(\omega)=\varepsilon_{\infty}+\left(\varepsilon-\varepsilon_{\infty}\right) \sum_{k} \frac{g_{k}}{1-\omega^{2} \tau_{k}^{2}}
$$

where $\tau_{k}$ is the characteristic relaxation time for the $k^{\text {th }}$ mechanism, $\varepsilon_{\infty}$ is the permittivity limit for infinitely fast processes, $\left\{g_{k}\right\}$ is the relative weight of the $k^{\text {th }}$ mechanism at which we have $\sum g_{k}=1,\left\{g_{k}\right\}$ and $\left\{\tau_{k}\right\}$ are the experimental values known for many wide-spread solvents.

At the absorption of emission of photons with the subsequent electronic transition, when the electron density of solute charges rapidly changes, we may assume $\varepsilon_{\mathrm{f}}=\varepsilon_{\mathrm{opt}}=n^{2}$, where $n$ is the refractive index of the solvent and $\varepsilon_{\mathrm{opt}}$ is the optical permittivity. This means that only solvent electrons can move to stay in equilibrium with a solute, while the molecular motion of a solvent are frozen during the process. In the TD-DFT calculations of the vertical electronic 
transitions, the equality of the permittivities $\varepsilon_{\mathrm{f}}=\varepsilon_{\mathrm{opt}}$ is taken in the conventional techniques. On the other hand, the solvent charge can be divided into the fast and slow components in order to reproduce a part of the solvation reaction field, which is always in equilibrium with a solute and the frozen part, respectively.

To check the effect of the solvent, dianions with four water molecules were calculated in the explicit form. Water was added opposite to oxygen atoms in rings 1,2, and 4 (see the structure in Table 1), so hydrogen was directed to the oxygen atom of the dianion. Then, the equilibrium geometry in the ground state was established and, based on this geometry, the absorption spectrum was calculated. The calculation showed that adding water in the explicit form does not significantly improve the results (Table 2). Therefore, adding explicit water is a possible way to improve the results. However, the drawback is overestimating the solvent effects because of ignoring the heat motion of a solvent, which could weaken the solvent bonding as compared with the cluster models. In the continuum model, this problem does not arise, although some solvent-solute charge transfer effects are ignored. The agreement between our results and the experiment indicates that these effects in the system under study are apparently weak.

Figure 1 shows the HOMO orbitals of all the calculated structures for the ground state $\mathrm{S}_{0}$ from which the quantum is absorbed and the LUMO orbital for the fluorescent state $\mathrm{S}_{1}^{*}$ from which molecules emit. It can be seen that the HOMO orbital have the $\pi$ bonding character for carbon atoms and the non-bonding character for oxygen and halogen atoms. The LUMO orbitals are also localized on the tricycle and have the same nature. At the transition from the HOMO to LUMO orbital, the electron density does not migrate; consequently, the charge transfer processes do not occur. All the transitions are characterized by the high (over unity) oscillator strength, which speaks about the locality of the optical transitions. All the calculated structures change their atomic structure at the transition to the equilibrium excited state. The greatest difference between the molecular geometry in the ground and excited states is observed for the erythrosin B dianion, in which the carboxyl group $\mathrm{COO}^{-}$is rotated relative to monocycle 1 , which, in turn, rotates relative to the tricycle. This can explain the fact that the fluorescence of the erythrosine B dianion has the largest wavelength.
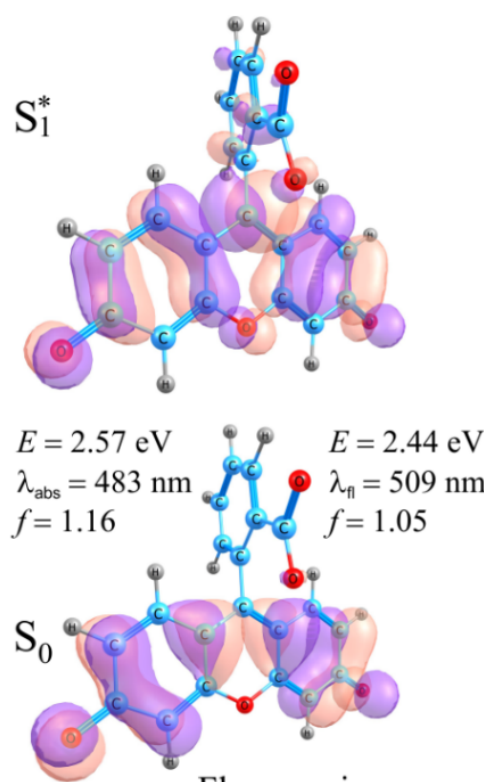

Fluorescein
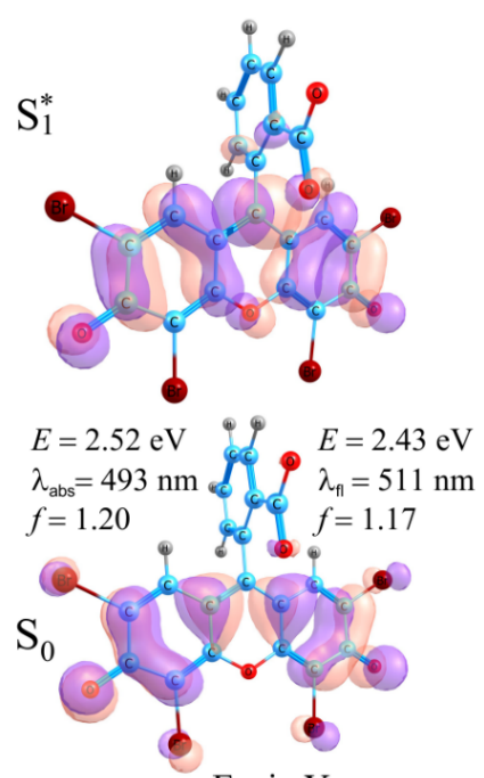

Eosin $\mathrm{Y}$
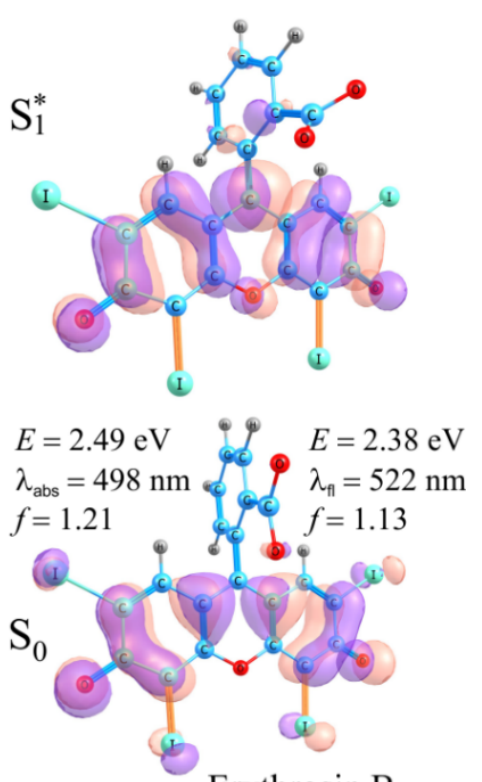

Erythrosin B

Figure 1. Pairs of the occupied HOMO molecular orbitals involved in the excitation in the ground state geometry and the virtual LUMO molecular orbitals involved in the emission in the excited state geometry. Excitation energies $E$, absorption $\lambda_{\text {abs }}$ and fluorescence wavelength $\lambda_{\mathrm{fl}}$, and oscillator strengths $f$ are shown. $\mathrm{S}_{0}$ and $\mathrm{S}_{1}^{*}$ corresponds to the equilibrium ground and excited states, respectively. 


\section{CONCLUSIONS}

Thus, the calculations at the B3LYP theory level showed that dianions of the fluorescein dyes (fluorescein, eosin Y, and erythrosin B) in the ground state can be presented in the form of a single spatial conformer. The effect of the solvent (water) on partial charges of oxygen atoms consists in a slight increase in the negative charge in the range of 0.04-0.09e. The positions of the spectra of the investigated dyes are affected by the basis set of the calculation and account for a medium and the solvent relaxation. All these factors, to different extents, lead to the red spectral shift of the absorption and fluorescence spectra. It was demonstrated that the account for a solvent in the explicit form causes a slight spectral shift, while the greatest effect of the solvent and the best agreement with the experimental data are obtained using the nonequilibrium solvation $\mathrm{PCM}^{*}$ method. The charge distributions on the chromophore group of fluorescein and its halogenated derivatives are essentially different, which forecasts the variation in the spectral properties of these dyes at the change in their ionic state.

\section{ACKNOWLEDGMENTS}

This work was supported by the Russian Foundation for Basic Research, project 19-02-00450.

\section{REFERENCES}

[1] Zheng, H., Zhan, X.-Q., Bian, Q.-N., and Zhang, X.-J., Advances in modifying fluorescein and rhodamine fluorophores as fluorescent chemosensors, Chem. Commun. 49, 429-447 (2013).

[2] Johnson, I. D., Practical considerations in the selection and application of fluorescent probes, in [Handbook for Biological Confocal Microscopy], Springer, New York, 353-367 (2006).

[3] Hilderbrand, S. A., Labels and probes for live cell imaging: overview and selection guide, Methods Mol. Biol. $591,17-45(2010)$.

[4] Tomasi, J., Mennucci, B., and Cammi, R., Quantum mechanical continuum solvation models, Chem. Rev. 105, 2999-3094 (2005).

[5] Slyusareva, E., Tomilin F., Sizykh A., The effect of halogen substitution on the structure and electronic spectra of fluorone dyes, Optics and Spectroscopy 112, 729-737 (2012)

[6] Cossi, M. and Barone, V., Time-dependent density functional theory for molecules in liquid solutions, J. Chem. Phys. 115, 4708-4717 (2001).

[7] Becke, A. D., Density-functional thermochemistry. The role of exact exchange, J. Chem. Phys. 98, 5648-5652 (1993).

[8] Lee, C., Yang, W., and Parr, R. G., Development of the Colle-Salvetti correlation-energy formula into a functional of the electron density, Phys. Rev. 37, 785-789 (1988).

[9] Gross, E. K. U. and Kohn W. Time-dependent density-functional theory, Ad. Quantum Chem. 21, 255-291 (1990).

[10] Casida, M. E., Time-dependent density functional response theory for molecules, [Recent Advances in Density Functional Theory], Vol. I, World Scientific, Singapore (1995).

[11] Schmidt, M. W., Baldridge, K. K., Boatz, J. A., Elbert, S. T., Gordon, M. S., Jensen, J. H., Koseki, S., Matsunaga, N., Nguyen, K. A., Su, S. J., Windus, T. L., Dupuis, M., and Montgomery J. A., General atomic and molecular electronic structure system, J. Comput. Chem. 14, 1347-1363 (1993).

[12] Schuchardt, K. L., Didier, B. T., Elsethagen, T., Sun, L., Gurumoorthi, V., Chase, J., Li, J., Windus, T. L., Basis set exchange: a community database for computational sciences, J. Chem. Inf. Model. 47, 1045-1052 (2007).

[13] Peach, M. J. G., Benfield, P., Helgaker, T., Tozer, D. J. Excitation energies in density functional theory: an evaluation and a diagnostic test, J. Chem. Phys. 128, 044118 (2008). 